\title{
Millimeter-wave Imaging of Pulsar Wind Nebulae
}

\author{
Douglas C.-J. Bock \\ Radio Astronomy Laboratory, University of California, 601 Campbell \\ Hall, Berkeley, CA 94720, USA \\ Bryan M. Gaensler \\ Harvard-Smithsonian Center for Astrophysics, 60 Garden Street MS-6, \\ Cambridge, MA 02138, USA
}

\begin{abstract}
We have observed the supernova remnants G16.7+0.1 and G29.7-0.3 at millimeter wavelengths with the Berkeley-Illinois-Maryland Association array, measuring total flux densities of the pulsar wind nebulae in the remnants. Our results imply a break at $\sim 50 \mathrm{GHz}$ in the spectrum of the PWN in G29.7-0.3, lower than previously estimated. The PWN in G16.7+0.1 either has a break $<100 \mathrm{GHz}$, or a steep radio spectrum.
\end{abstract}

\section{Introduction}

At radio frequencies, pulsar wind nebulae (PWNe) typically have flat spectra $-0.3<\alpha<0\left(S_{\nu} \propto \nu^{\alpha}\right)$, but in the X-ray band we generally see $\alpha<-1$. It is therefore commonly presumed that PWNe have at least one spectral break at intermediate wavelengths. Such breaks are expected from theoretical considerations, and result from a combination of synchrotron losses and the time-evolution of the pulsar's changing energy output. Locating these breaks gives a key insight into the physical conditions of the pulsar wind: in PWNe powered by young $(<5 \mathrm{kyr})$ pulsars, the standard synchrotron loss break can be used to infer directly the nebular magnetic field strength (e.g. Frail et al. 1996), while spatial variations in the break energy can be used to identify and map out the processes of particle diffusion and radiative losses within the flow (Bock, Wright \& Dickel 2001).

Most flux density measurements of PWNe to date have been in the Xray and radio bands. To pin down the break frequencies it is necessary to measure flux densities in the part of the spectrum near the spectral breaks, i.e. at millimeter and infrared wavelengths. Here we report on observations of two PWNe (in the supernova remnants G16.7+0.1 and G29.7-0.3) selected for being sufficiently bright and compact to allow imaging with millimeter interferometry. A third PWN observed, in G11.2-0.3, was not sufficiently well-imaged to allow accurate flux density measurements. 


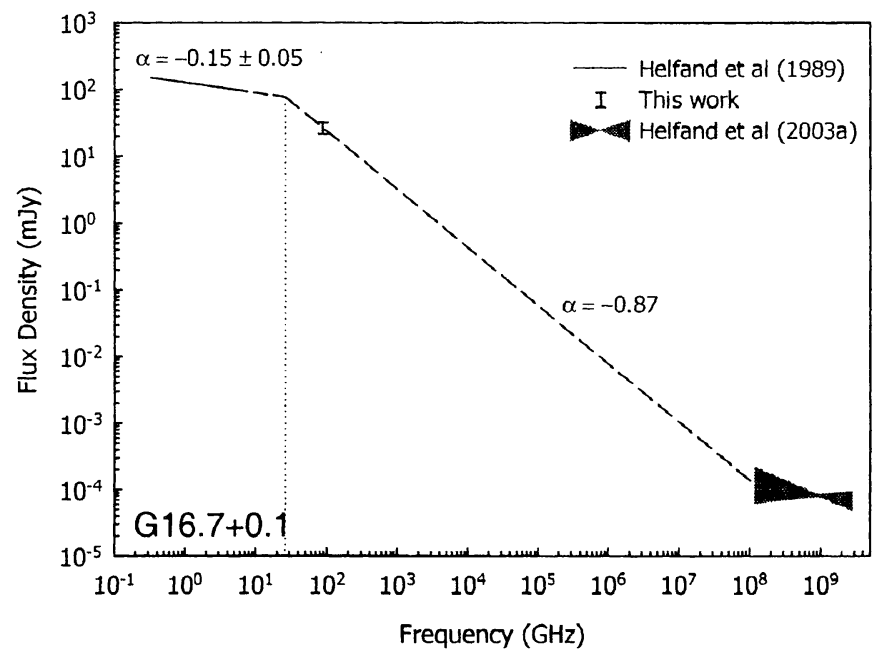

Figure 1. Integrated spectrum of the PWN in G16.7+0.1.

\section{Observations}

Mosaiced observations were obtained at $88.6 \mathrm{GHz}$ using the BIMA array during 2002 and 2003. To achieve suitable $u v$ coverage, we used the technique of multifrequency synthesis over both $800 \mathrm{MHz}$ sidebands of the local oscillator. Using 90- $\mathrm{GHz}$ quarter-wave plates, we measured only the left circular polarization (IEEE) in the assumption that circularly-polarized emission from the sources would be negligible. The antenna gains were determined from observations of planets at short antenna separations; the uncertainty in the flux density scale is about $10 \%$. Imaging was done with the MIRIAD software package, using Steer-Dewdney-Itoh (CLEAN-based) deconvolution.

\section{Results and Discussion}

\subsection{G16.7+0.1}

We have just detected emission from the PWN in this remnant: there is a $3.5 \sigma$ peak of $14 \mathrm{mJy}^{\text {beam }}{ }^{-1}$ at $08^{\mathrm{h}} 20^{\mathrm{m}} 57.2-14^{\circ} 19^{\prime} 34^{\prime \prime}(\mathrm{J} 2000)$. An elliptical Gaussian fitted to the feature has a size of $51^{\prime \prime} \times 20^{\prime \prime}$ (at position angle $6^{\circ}$ ), and an integrated flux density of $27 \mathrm{mJy}$. The size and position are consistent with the measurements of Helfand et al. (1989). Modeling indicates that we should have measured more than $90 \%$ of the emission from this object, and we consider this a reliable measurement of the flux density of the PWN at this frequency.

A spectrum of the PWN in G16.7+0.1 is shown in Figure 1. There are few measurements available of the flux density of this PWN: the simple X-ray to radio spectral index is shown. The radio and $\mathrm{X}$-ray spectral index measurements imply at least two breaks between the regimes. Our measurement indicates that there is a break at less than $90 \mathrm{GHz}$, with the exact frequency set by the shape of the spectrum between millimeter and X-ray wavelengths. 


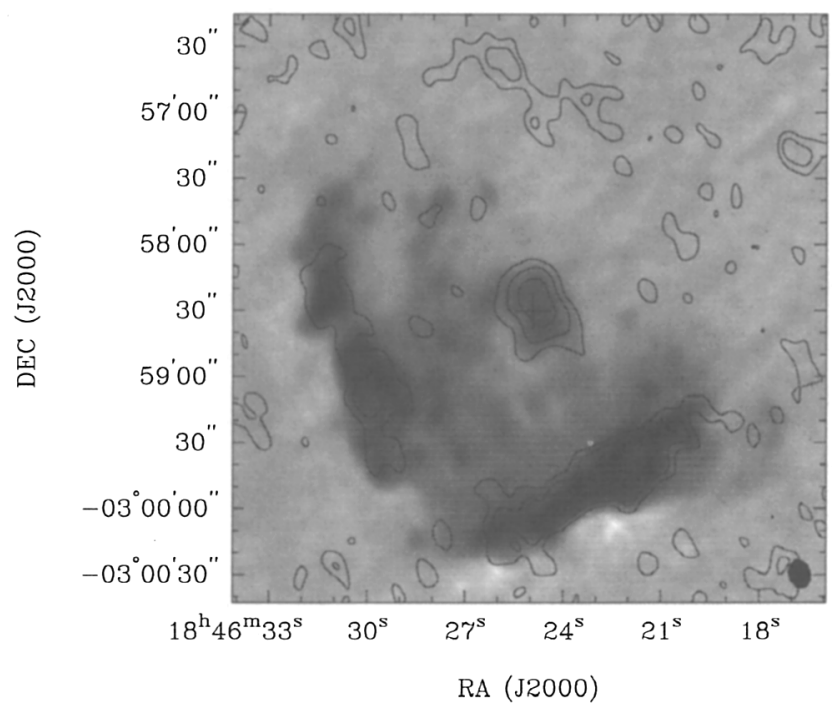

Figure 2. Image of G29.7-0.3 at $89 \mathrm{GHz}$ (contours) and $1.4 \mathrm{GHz}$ (logarithmic grayscale; Helfand et al. 2003b). The contours are at 2, $5,10,20 \mathrm{mJy} \mathrm{beam}^{-1}$. The synthesized beam at $89 \mathrm{GHz}$ is shown at the lower right.

\subsection{G29.7-0.3 (Kesteven 75)}

The BIMA image of G29.7-0.3 is presented in Figure 2. For comparison, the 1.4 $\mathrm{GHz}$ image of Helfand, Collins \& Gotthelf (2003) is shown also. In the figure, both the PWN (at the image center) and partial shell (to the south-east) can easily be seen at each frequency. As expected, the PWN is relatively brighter than the shell at the higher frequency, i.e., it has a flatter spectral index. Note that the PWN is central in the corresponding X-ray image (Helfand et al. 2003b), which also shows the northern extent of the SNR's shell.

We have measured an $89 \mathrm{GHz}$ flux density for the PWN of $80 \mathrm{mJy}$ in $0.6 \operatorname{arcmin}^{2}$ (within the "0 mJy beam ${ }^{-1}$ contour"), taking into account a local background within the SNR shell (probably due to imperfect deconvolution) of approximately $-36 \mathrm{mJy} \operatorname{arcmin}^{-2}$. At the nearby frequency of $84 \mathrm{GHz}$, the observations of Salter et al. (1989) imply an integrated flux density of $159 \mathrm{mJy}$, inconsistent with the present data. However, the earlier observations were made using a single dish with a beam of $76^{\prime \prime} \times 70^{\prime \prime}$, substantially larger than the characteristic source size $\left(26^{\prime \prime} \times 17^{\prime \prime}\right)$ measured by Hunt et al. (as reported by Salter et al. 1989). It seems likely that Salter et al. also measured some shell emission. For similar reasons the $32 \mathrm{GHz}$ measurement of Morsi \& Reich (1987) may best be considered an upper limit to the emission from the PWN. The advantage of the present interferometric measurement is that it would have filtered out any contaminating emission from the SNR's shell, and should be a more reliable determination of the flux density attributable to the PWN alone.

A comparison with the published measurements of the PWN flux density at other frequencies (Fig. 3) indicates that there is probably a spectral break 


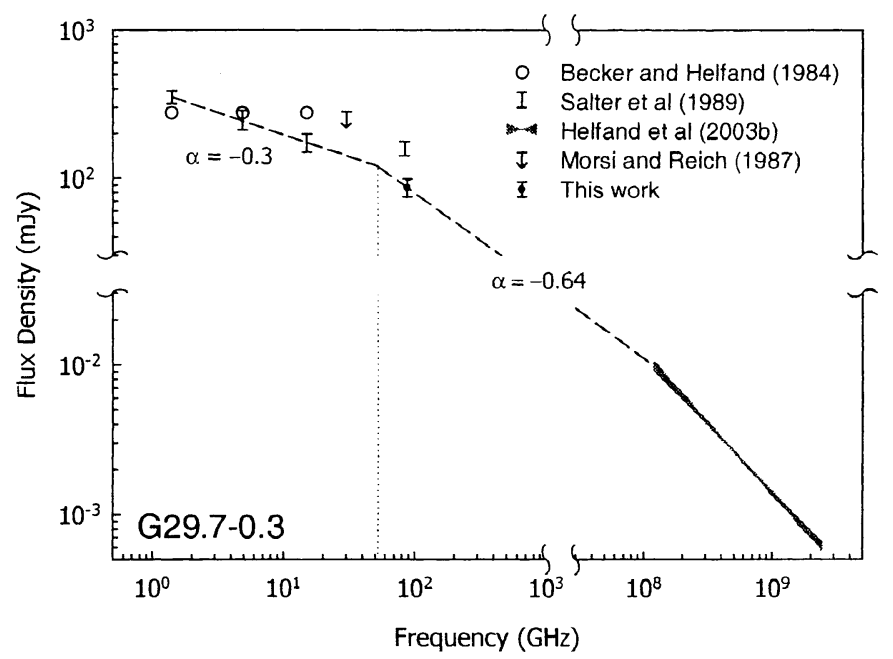

Figure 3. Integrated spectrum of the PWN in G29.7-0.3.

below $90 \mathrm{GHz}$. The low-frequency measurements of Salter et al. (1989) constrain this break to near $50 \mathrm{GHz}$, with the exact value dependent on the shape of the spectrum above $90 \mathrm{GHz}$. However, the earlier measurements of Becker \& Helfand (1984) imply an even lower break frequency.

The nebula may have a different spectrum on either side of the pulsar (marked with a cross in Fig. 2). At $1.4 \mathrm{GHz}$ the PWN is brightest to the north of the pulsar, while at $89 \mathrm{GHz}$ and in X-rays the southern lobe is brighter. A more detailed analysis of the $1.4 \mathrm{GHz}$ and $\mathrm{X}$-ray data should clarify the situation.

Acknowledgments. We thank E. Gotthelf and D. Helfand for making available their data in electronic form, and M. Wright for useful discussions. The BIMA array is operated by the University of California, Berkeley, University of Illinois, and University of Maryland with support from the National Science Foundation.

\section{References}

Becker, R. H., \& Helfand, D. J. 1984, ApJ, 283, 154

Bock, D. C.-J., Wright, M. C. H., \& Dickel, J. R. 2001, ApJ, 561, L203

Frail, D. A., Giacani, E. B., Goss, W. M., \& Dubner, G. 1996, ApJ, 464, L165

Helfand, D. J., Velusamy, T., Becker, R. H., \& Lockman, F. J. 1989, ApJ, 341, 151

Helfand, D. J., Agüeros, M. A., \& Gotthelf, E. V. 2003a, ApJ, 592, 941

Helfand, D. J., Collins, B. F., \& Gotthelf, E. V. 2003b, ApJ, 582, 783

Morsi, H. W., \& Reich, W. 1987, A\&AS, 71, 189

Salter, C. J., Reynolds, S. P., Hogg, D. E., Payne, J. M., \& Rhodes, P. J. 1989, ApJ, 338, 171 